\title{
CORRECTION
}

W) Check for updates

Cite this: RSC Adv., 2020, 10, 9499

DOI: $10.1039 /$ dOra90020f

rsc.li/rsc-advances

\section{Correction: Chemical fingerprinting and quantitative monitoring of the doping drugs bambuterol and terbutaline in human urine samples using ATR-FTIR coupled with a PLSR chemometric tool}

\author{
Faisal K. Algethami, (D) a Sherif M. Eid, (iD *b Khadiga M. Kelani, ${ }^{\text {cd }}$ \\ Mohamed R. Elghobashy (D) bc and Mohamed K. Abd El-Rahman (D) ${ }^{c}$
}

Correction for 'Chemical fingerprinting and quantitative monitoring of the doping drugs bambuterol and terbutaline in human urine samples using ATR-FTIR coupled with a PLSR chemometric tool' by Faisal K. Algethami et al., RSC Adv., 2020, 10, 7146-7154.

The authors regret that one of the affiliations (affiliation $a$ ) was incorrectly shown in the original manuscript. The corrected list of affiliations is as shown in this Correction article.

The Royal Society of Chemistry apologises for these errors and any consequent inconvenience to authors and readers.

\footnotetext{
${ }^{a}$ Chemistry Department, Faculty of Science, Imam Mohammad Ibn Saud Islamic University, Riyadh, Saudi Arabia
}

${ }^{b}$ Analytical Chemistry Department, Faculty of Pharmacy, October 6 University, 6 October City, Giza, Egypt. E-mail: Sheriefmohammed@o6u.edu.eg; Sheriefo55@icloud.com; Tel: $+201111609539$

'Analytical Chemistry Department, Faculty of Pharmacy, Cairo University, El-Kasr El-Aini Street, ET-11562 Cairo, Egypt

${ }^{d}$ Analytical Chemistry Department, Faculty of Pharmacy, Modern University for Technology and Information, Cairo, Egypt 